EPJ Web of Conferences 66, 02057 (2014)

DOI: $10.1051 /$ epjconf/ 20146602057

(C) Owned by the authors, published by EDP Sciences, 2014

\title{
Study of shape transition in the neutron-rich Os isotopes
}

P.R. John ${ }^{1,2, a}$, V. Modamio ${ }^{3}$, J.J. Valiente-Dobón ${ }^{3}$, D. Mengoni ${ }^{1,2}$, A. Gottardo ${ }^{3}$, D. Bazzacco ${ }^{2}$, S. Lunardi ${ }^{1,2}$, T. Alexander ${ }^{4}$, G. de Angelis ${ }^{3}$, N. Ashwood ${ }^{5}$, M. Barr ${ }^{5}$, P.G. Bizzeti ${ }^{6,7}$, A.M. BizzetiSona $^{6,7}$, S. Bottoni ${ }^{8,9}$, M. Bowry ${ }^{4}$, A. Bracco ${ }^{8,9}$, F. Browne ${ }^{10}$, M. Bunce ${ }^{4}$, A. Gadea ${ }^{11}$, F. Camera ${ }^{8,9}$, L. Corradi ${ }^{3}$, F.C.L. Crespi ${ }^{8,9}$, E. Farnea ${ }^{2}$, E. Fioretto ${ }^{3}$, Tz. Kokalova ${ }^{5}$, W. Korten ${ }^{12}$, A. Kuşoğlu' ${ }^{13}$, S. Lenzi ${ }^{1,2}$, S. Leoni ${ }^{8,9}$, C. Michelagnoli ${ }^{1,2}$, T. Mijatović ${ }^{14}$, G. Montagnoli ${ }^{1,2}$, D. Montanari ${ }^{1,2}$, D.R. Napoli ${ }^{3}$, Zs. Podolyák ${ }^{10}$, G. Pollarolo ${ }^{15,16}$, F. Recchia ${ }^{1,2}$, O.J. Roberts ${ }^{10}$, E. Şahin ${ }^{3}$, M.-D. Salsac ${ }^{12}$, F. Scarlassara ${ }^{1,2}$, A.M. Stefanini ${ }^{3}$, S. Szilner ${ }^{14}$, C.A. Ur ${ }^{2}$, J. Walshe ${ }^{5}$, and C. Wheldon ${ }^{5}$

${ }^{1}$ Dipartimento di Fisica e Astronomia, Università degli Studi di Padova, Padova, Italy

${ }^{2}$ INFN Sezione di Padova, Padova, Italy

${ }^{3}$ INFN Laboratori Nazionali di Legnaro, Legnaro, Italy

${ }^{4}$ Department of Physics, University of Surrey, Guildford, United Kingdom

${ }^{5}$ School of Physics and Astronomy, University of Birmingham, Birmingham, United Kingdom

${ }^{6}$ Dipartimento di Fisica, Università degli Studi di Firenze, Firenze, Italy

${ }^{7}$ INFN Sezione di Firenze, Firenze, Italy

${ }^{8}$ Dipartimento di Fisica, Università degli Studi di Milano, Milano, Italy

9 INFN Sezione di Milano, Milano, Italy

${ }^{10}$ University of Brighton, Brighton, United Kingdom

${ }^{11}$ Instituto de Física Corpuscular, CSIC, Valencia, Spain

${ }^{12}$ CEA/Saclay, IRFU/Service de Physique Nucleaire, Gif-sur-Yvette, France

${ }^{13}$ Istanbul Üniversitesi, İstanbul, Turkey

${ }^{14}$ Institut Ruđer Bošković, Zagreb, Croatia

${ }^{15}$ Dipartimento di Fisica, Università degli Studi di Torino, Torino, Italy

${ }^{16}$ INFN Sezione di Torino, Torino, Italy

\begin{abstract}
.
The neutron-rich isotopes of tungsten, osmium and platinum have different shapes in their ground states and present also shape transitions phenomena. Spectroscopic information for these nuclei is scarce and often limited to the gamma rays from the decay of isomeric states. For the neutron-rich even-even osmium isotopes ${ }^{194} \mathrm{Os}$ and ${ }^{198} \mathrm{Os}$, a shape transition between a slightly prolate deformed to an oblate deformed ground state was deduced from the observed level schemes. For the even-even nucleus lying in between, ${ }^{196} \mathrm{Os}$, no gamma ray transition is known. In order to elucidate the shape transition and to test the nuclear models describing it, this region was investigated through gamma-ray spectroscopy using the AGATA demonstrator and the large acceptance heavy-ion spectrometer PRISMA at LNL, Italy. A two-nucleon transfer from a ${ }^{198} \mathrm{Pt}$ target to a stable ${ }^{82} \mathrm{Se}$ beam was utilized to populate medium-high spin states of ${ }^{196} \mathrm{Os}$. The analysis method and preliminary results, including the first life-time measurement of isomeric states with AGATA, are presented.
\end{abstract}

ae-mail: philipp.john@pd.infn.it

This is an Open Access article distributed under the terms of the Creative Commons Attribution License 2.0, which permits unrestricted use, distribution, and reproduction in any medium, provided the original work is properly cited. 


\section{Introduction}

The nuclei with $A \approx 190$ between Hf and Pt exhibit a great variety of nuclear phenomena, including Kisomeric states, triaxiality, different shapes in their ground states and shape transitions between them. The ground states of the lighter isotopes are prolate deformed. When going to the more neutron-rich isotopes a shape transition occurs and the ground state becomes oblate deformed. The spherical shape is believed to be restored at the $N=126$ shell closure.

Experimentally, these nuclei are very difficult to populate and therefore the spectroscopic information is rather scarce. Multi-nuclei transfer and fragmentation reactions are the only possible methods to reach such nuclei. In the present experiment we used a multi-nucleon transfer reaction together with the large-acceptance magnetic spectrometer PRISMA [1] and the gamma-ray spectrometer AGATA $[2,3]$ in order to study the nuclear properties in this interesting nuclear region.

\section{Experiment}

A ${ }^{82}$ Se beam impinging on a $2 \mathrm{mg} / \mathrm{cm}^{2}$ self-supporting ${ }^{198} \mathrm{Pt}$ target was used to populate the Os nuclei. The beam, with an energy of $426 \mathrm{MeV}$ and an intensity of around $2-3 \mathrm{pnA}$, was provided by the Tandem-ALPI accelerator complex at LNL, Italy. The beam-like fragments were detected by the PRISMA spectrometer, placed at the grazing angle of the beam-like recoils $\left(57^{\circ}\right)$. The AGATA demonstrator, placed at a distance of $15.5 \mathrm{~cm}$ from the target, was used for detecting the emitted gamma rays. Additionally, an array of four DANTE [4] micro-channel plate heavy-ion detectors was placed around a cone of $58^{\circ}$ with respect to the beam axis, to detect the beam-like as well as the target-like recoils.

Figure 1 (left) shows the sketch of the experimental setup where the kinematics of the reaction is shown. The beam-like ions enter the PRISMA spectrometer after an average time of flight of around $10 \mathrm{~ns}$, while the binary partner of the beam-like recoil, gets implanted in one of the DANTE detectors or the target chamber. Therefore, delayed gamma rays in coincidence with PRISMA can be observed only for the target-like ions. The time of flight for these recoils is around $15 \mathrm{~ns}$, and consequently the sensitivity for isomeric states gets reduced for lifetimes shorter than $15 \mathrm{~ns}$.
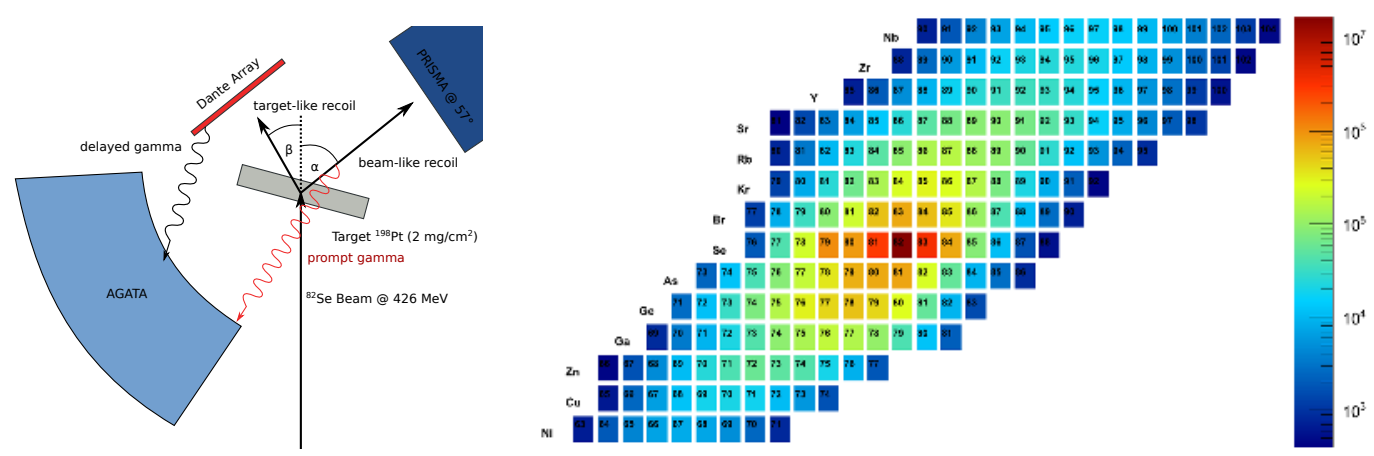

Figure 1. (left) Sketch of the experimental setup and of the reaction kinematics. The dimensions are not in scale. (right) Measured yield of the beam-like recoils for the ${ }^{82} \mathrm{Se}$ on ${ }^{198} \mathrm{Pt}$ multi-nucleon transfer reaction at a beam energy of $426 \mathrm{MeV}$. 


\section{Data Analysis}

The identification of the beam-like recoils is performed event-by-event by measuring their atomic charge and mass. The extracted yield is shown in Figure 1. The Doppler correction for the beam-like recoils uses the velocity vector measured by PRISMA and the first interaction point of the tracked gamma ray in AGATA. The velocity vector of the heavier target-like recoil is reconstructed using relativistic two-body reaction kinematics. The energy loss in the target for each recoil is calculated for every trajectory using the Northcliffe-Schilling [5] approximation. It is assumed that the reaction occurs in the centre of the target and particle evaporation is neglected. Doppler-corrected spectra for the beam as well as the target ions are shown in Figure 2. The FWHM of the $729.1 \mathrm{keV}\left(6_{1}^{+}\right) \rightarrow 4_{1}^{+}$ transition of ${ }^{198} \mathrm{Pt}$ is $4.4 \mathrm{keV}(0.61 \%)$.

With traditional HPGe detector arrays the time between the heavy-ion detector and the different germanium detectors is measured by using TACs or TDCs. In AGATA the reconstructed trajectory of the gamma ray can span over more than one segment and crystal. Hence, the time of the interaction of each gamma ray is deduced from its first interaction point, as reconstructed by the tracking algorithm OFT [8]. The time of each interaction is calculated on a sub-timestamp level using the recorded traces. In order to improve the resolution of the prompt peak in coincidence with PRISMA, the data has been corrected by the time of flight of the ions entering PRISMA. A second correction is applied to account for offsets in time with respect to different sections of the focal plane detector.

Previous HPGe detector arrays coupled to a magnetic spectrometer such as, CLARA [9] and EXOGAM [10] have anti-Compton shields and collimators in front of the detectors to achieve a good peak-to-total ratio and to suppress the background. Hence, the efficiency drops for gamma rays, not emitted at the target position. AGATA instead does not have any anti-Compton shields and reconstructs the Compton-scattering among the crystals by using a tracking algorithm. Therefore, the detection efficiency of gamma rays emitted by the ions implanted in the target chamber is significantly higher than with traditional arrays.

In Figure 3 (left) the matrix of the corrected time difference of PRISMA and AGATA versus the tracked gamma energy is shown. In order to study and verify $5_{1}^{+}$the isomeric state known in ${ }^{198} \mathrm{Au}$, the matrix is gated on all arsenic isotopes identified in PRISMA with a mass smaller than ${ }^{82} \mathrm{As}$. The two transitions visible at $215 \mathrm{keV}$ and $97 \mathrm{keV}$ belong to the deexcitation of the $5_{1}^{+}$isomer in ${ }^{198} \mathrm{Au}$. This

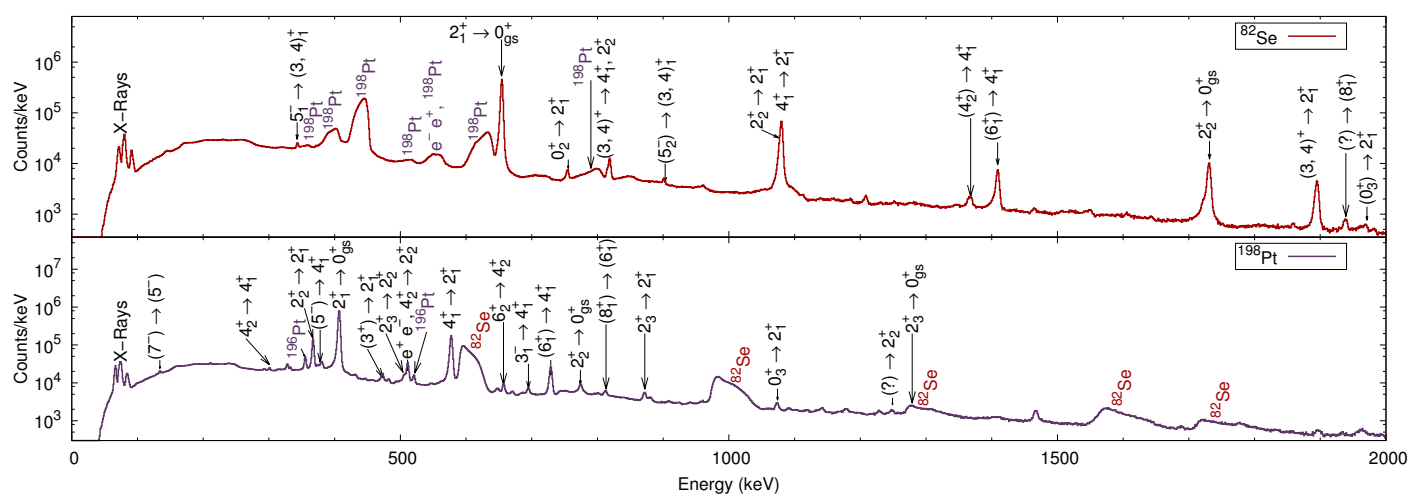

Figure 2. Doppler-corrected gamma energy spectra for ${ }^{82} \mathrm{Se}$ (top) and the binary partner ${ }^{198} \mathrm{Pt}$ (bottom). The transitions are marked tentatively according to the previously reported data $[6,7]$. 
isomer was reported in the sixties and seventies [11-14] with a half life ranging from $118(8) \mathrm{ns}$ [14] to $128(15) \mathrm{ns}$ [11]. In our experiment the half life was measured to be 107 (6) ns. It was obtained by a least-square fit of the exponential decay after subtracting the background, see Figure 3 (right). This is the first life-time measurement of isomeric states with AGATA.
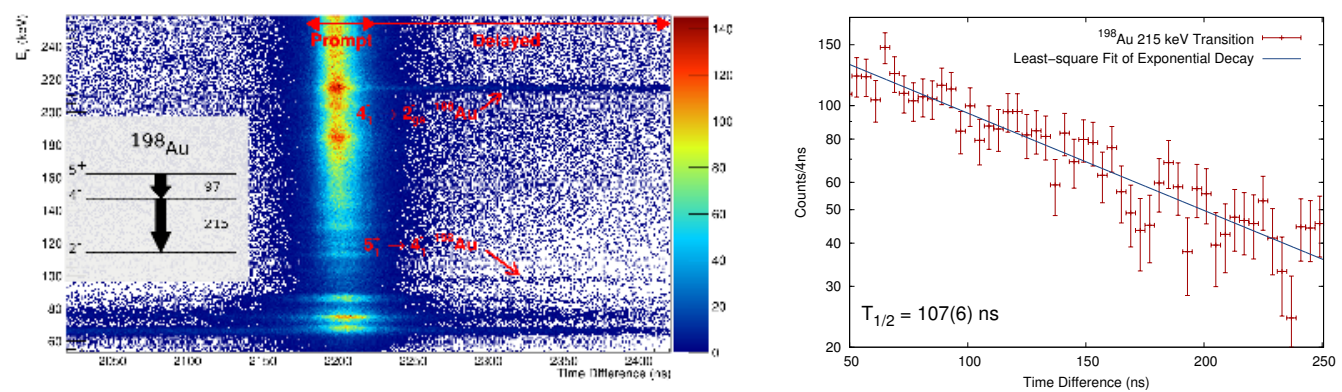

Figure 3. (left) Matrix of the corrected time difference of PRISMA and AGATA versus the tracked gamma energy gated on all arsenic isotopes with a mass of 82 and smaller. The time axis has an arbitrary offset. (right) Exponential least-square fit of the decay curve of the $215 \mathrm{keV}$ transition with the half-life derived for the $5_{1}^{+}$isomer in ${ }^{198} \mathrm{Au}$.

\section{Conclusions and Outlook}

The gamma-ray data obtained in this multi-nucleon-transfer experiment are promising and they will help to elucidate the shape transition occurring in the $A \approx 190$ region. In addition to the Os isotopes, a great variety of other reaction channels were populated. In particular, the binary partner of ${ }^{196} \mathrm{Os}$, ${ }^{84} \mathrm{Kr}$, was detected with a high yield.

\section{References}

[1] A. Stefanini et al., Nucl. Phys. A 701, 217 (2002)

[2] S. Akkoyun et al., NIM A 668, 26 (2012)

[3] A. Gadea et al., NIM A 654, 88 (2011)

[4] A. Gottardo et al., Nucl. Phys. A 805, 606 (2008)

[5] L. Northcliffe, R. Schilling, Atomic Data and Nuclear Data Tables 7, 233 (1970)

[6] H. Xiaolong, Nucl. Data Sheets 110, 2533 (2009)

[7] J. Tuli, Nucl. Data Sheets 98, 209 (2003)

[8] A. Lopez-Martens et al., NIM A 533, 454 (2004)

[9] A. Gadea et al., Euro. Phys. J. A 20, 193 (2003)

[10] F. Azaiez, Nucl. Phys. A 654, 1003c (1999)

[11] M. Bonitz, Nucl. Phys. A 118, 478 (1968)

[12] K. Löbner et al., Z. für Physik 235, 254 (1970)

[13] J. Mirza et al., Z. für Physik 272, 175 (1975)

[14] A. Pakkanen et al., Nucl. Phys. A 206 (1973) 